Polymer Journal, Vol. 23, No. 5, pp 593-601 (1991)

\title{
Lipid Bilayer Membranes Containing Glycopeptides as Receptor Model
}

\author{
Y. IMANISHI* and S. KIMURA \\ Department of Polymer Chemistry, Faculty of Engineering, Kyoto University, \\ Yoshida Honmachi, Sakyo-ku, Kyoto 606, Japan
}

(Received February 21, 1991)

\begin{abstract}
A number of glycopeptides were synthesized and investigated on their interactions with lipid bilayer membranes. Glycopeptides in which $\mathrm{N}$-acetylglucosamine and a tripeptide are connected by aliphatic chains of different lengths were distributed to liposomes and influenced the structure of lipid bilayer membrane upon crosslinking by lection. However, the tripeptide chain was too short to penetrate the lipid membrane to form an ion channel across the membrane. Next, glycopeptides in which maltose is connected to $\alpha$-helical polyalanines (DP 15 and 25) were synthesized. However, distribution of these glycopeptides to lipid bilayer membrane was very low. Finally, synthetic glycopeptides, Boc-(Ala-Aib) ${ }_{n}$-Lac, were distributed and oriented perpendicularly to the plane of lipid bilayer membrane. It was found that the glycopeptide with $n=4$ formed an aggregate in the membrane to form an ion channel upon interaction with lectin.

KEY WORDS Glycopeptide / Phospholipid Vesicle / Lectin / Crosslinking / Phase Separation / Ion Channel /
\end{abstract}

Glycoproteins in biological membranes play important roles in intra- and intercellular interactions. In order to obtain informations on the intra- and intercellular interactions, unilamellar vesicles containing synthetic glycopeptides were prepared. In these artificial cells, it was expected that glycopeptides could act as receptors responsive to biological signals transported by lectin, and that the biological signal should be converted to the variation of membrane properties and ion permeabilities across the membrane. In the present investigation, the effect of intervesicular or intravesicular crosslinking of glycopeptides by lectin on the incorporation of the glycopeptides into the membrane and, in particular, on the formation of an ion channel across the membrane was investigated.

\section{EXPERIMENTAL}

Glycopeptides as models for glycoproteins and peptides used in the present investigation are shown in Figure 1.

Glycopeptides in which N-acetylglucosamine (GlCNAC) and a tripeptide (Asp(OBzl)-Phe-Lys-OMe) substituted with a fluorescent probe (pyrene) are connected by aliphatic chains of different lengths were synthesized by the conventional liquid-phase method and are represented as $\mathrm{S}_{5}$ or $\mathrm{S}_{10}$. 
Y. IMANISHI and S. KiMURA
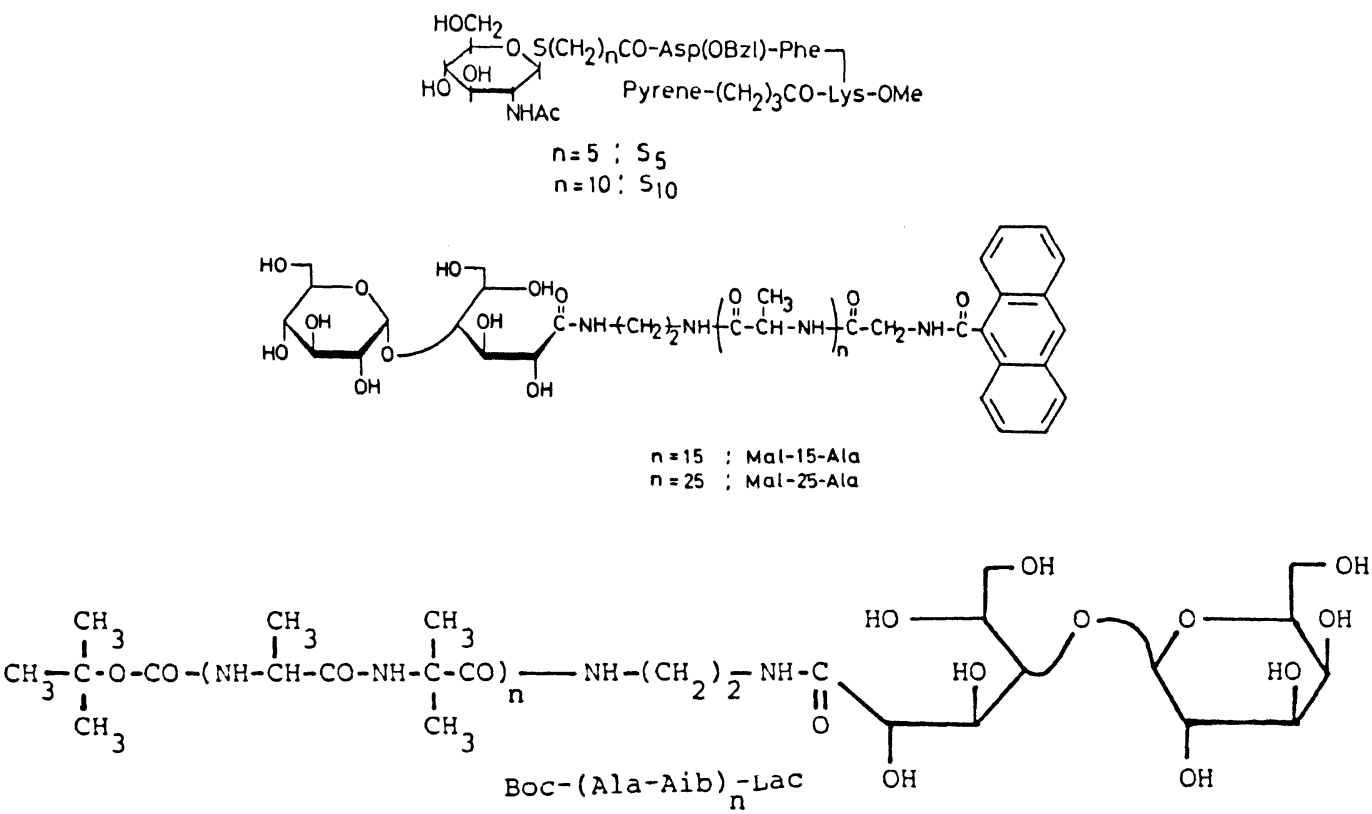

Figure 1. Molecular structures of glycopeptides synthesized in the present investigation.

Glycopeptides in which maltose is connected to $\alpha$-helical poly(Lalanine) carrying anthryl group at the amino terminal were synthesized by the polymerization of L-Ala NCA initiated by acetymaltonolactone ethylenediamine followed by acylation with $\mathrm{N}$-anthroylglycine at the amino terminal and deacetylation. 2 These glycopeptides are represented as Mal15-Ala or Mal-25-Ala.

Alamethicin-model peptides, $\operatorname{Boc}-(A l a-A i b) n-O M e(n=2,4,6,8)$, their anthrylated derivatives, $\operatorname{Boc}-\operatorname{Ser}\left(\mathrm{CH}_{2} \mathrm{Ant}\right)-(\mathrm{Ala}-\mathrm{Aib})_{n}-\mathrm{OMe}(\mathrm{n}=2,4,6,8,10)$, and their lactosylated derivatives, Boc-(Ala-Aib)n $-\operatorname{Lac}(n=2,4)$, were synthesized by the conventional liquid-phase method. $3^{\text {n }}$

The purity of all glycopeptides was verified by elemental analyses, IR, ${ }^{1} \mathrm{H}$ NMR, ${ }^{13} \mathrm{C}$ NMR, and TLC.

Small unilamellar vesicle (SUV) composed of 1,2-dipalmitoyl (or dimyristoyl)-sn-glycero-3-phosphocholine (DPPC or DMPC) or egg yolk lethicin was prepared by sonication of the lipid dispersion in a buffer solution (10 mM 2-[4-(2-hydroxymethyl)-1-piperazyl]ethanesulfonic acid (HEPES), $0.1 \mathrm{M} \mathrm{NaCl}$ or $\mathrm{KCl}, 0.1 \mathrm{mM}$ EDTA, $\mathrm{pH} 7.4$ ) and ultracentrifugation at $100000 \mathrm{~g}$. Lipid content was assayed by colorimetric method using phospholipase D (Diacolor, Toyobo, Japan).

Carboxyfluorescein ( $C F$ ) leakage from DPPC vesicles was measured according to the method reported by Barbet et al.4 Fluorescence excitation and monitoring wavelengths of $\mathrm{CF}$ were 470 and $515 \mathrm{~nm}$, respectively.

Aggregation of vesicles induced by the addition of wheat germ agglutinin (WGA) or concanavalin A (Con A) was monitored by the absorption at 360 $\mathrm{nm}$.

Fluorescence depolarization of fluorescent group connected to the peptides was measured on an MPF-4 fluorophotometer installed with an equipment as previously reported. ${ }^{1}$ Fluorescence excitation and monitoring wavelengths of 9-anthracenecarbonyl group of the glyco- 
peptides were 365 and $425 \mathrm{~nm}$, respectively.

Excitation wavelengths of 8-anilino-1-naphthalenesulfonate (ANS) and 1,6-diphenyl-1,3,5-hexatriene were 395 and $380 \mathrm{~nm}$, repectively.

\section{RESULTS AND DISCUSSION}

Phospholipid vesicles containing $\mathrm{S}_{5}$ or $\mathrm{S}_{10}$

$\mathrm{S}_{5}$ was soluble in HEPES buffer under the conditions of the fluorescence measurement to show reasonable fluorescence spectra. Addition of acrylamide, which is a water-soluble quencher, did not decrease the intensity of $S_{5}$ emission in the presence of DPPC vesicles, though it did so in the absence of vesicles. This means that the pyrenyl group of $\mathrm{S}_{5}$ in the presence of vesicles is buried in a hydrophobic core of lipid bilayer and not exposed to the water phase for quenching. The emission of $\mathrm{S}_{5}$ and $\mathrm{S}_{10}$ in the presence of DPPC vesicles was quenched more efficiently by 16-doxylstearic acid than by 5-doxylstearic acid, which means that the pyrenyl group is buried deeply in the membrane.

The addition of WGA to the $\mathrm{S}_{5}$-containing vesicles induced agglutination of vesicles as shown by the increased turbidity of the suspension. The aggregation of vesicles through the intervesicular bridge of WGA was dissociated by the addition of $N, N^{\prime}$-diacetylchitobiose reversibly. It was notable that GlCNAC was too weak to antagonize the aggregation of vesicles.

The emission intensity from pyrenyl excimer ( $I_{D}$ ) in the presence of vesicles remained almost constant above the phase-transition temperature. However, as the temperature went down below the phase-transition temperature, $I_{D}$ increased. The same phenomenon was observed with $\mathrm{S}_{10}$. The increase of $I_{D}$ below the phase-transition temperature is interpreted in terms of segregation of membrane into liquid-crystalline lipid regions and other regions where $S_{5}$ or $S_{10}$ is condensed. 5

Figure 2 shows the change in $I_{D} / I_{M}$ ( $I_{M}$ represents the emission intensity of pyrene monomer) of $S_{5}$ by the addition of WGA. As the WGA concentration added to the suspension was increased, the $I_{D} / I_{M}$ value increased, indicating intravesicular association of $\mathrm{S}_{5}$ molecules by the crosslinking of the glyco moiety with WGA. The crosslinking of $\mathrm{S}_{5} \mathrm{mo}^{-}$ lecules with WGA was enhanced below the phase-transition temperature. The intravesicular crosslinking of $\mathrm{S}_{5}$ induced by WGA above the phasetransition temperature was disrupted by the addition of GlcNAc as well as $N, N^{\prime}$-diacetylchitobiose, and the original distribution of $\mathrm{S}_{5}$ in the membrane was restored reversibly. However, below the phase-transition temperature the $I_{D} / I_{M}$ value was not decreased by the addition of $N, N^{\prime}$-diacetylchitobiose because of the immobilization of associated $\mathrm{S}_{5}$ molecules in a crystalline state of the membrane.

As shown in Figures 3 and 4 , the distribution of $\mathrm{S}_{5}$ into DPPC vesicles decreased the membrane fluidity in all cases. However, the fluidity of the hydrophobic

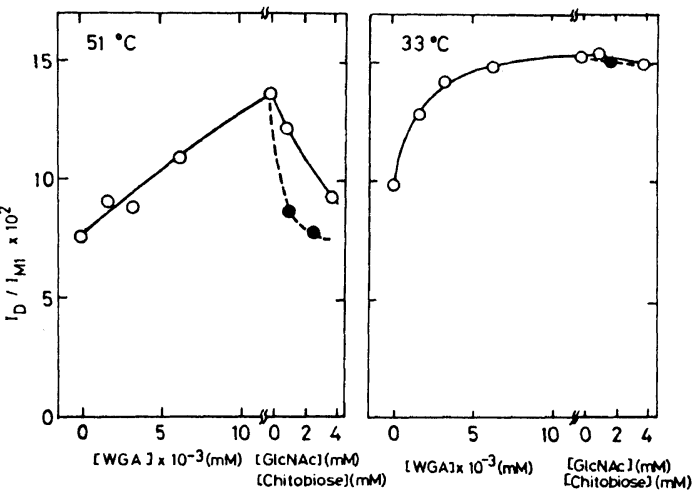

Figure 2. Change of $I_{D} / I_{M}$ of $S_{5}(1.89$ $x 10^{-2} \mathrm{mM}$ ) in the presence of DPPC vesicles $(1.0 \mathrm{mM})$ with the addition of WGA. The effects of the addition of GlCNAC ( 0 ) or N, N'-diacetylchitobiose (-) are shown together. 


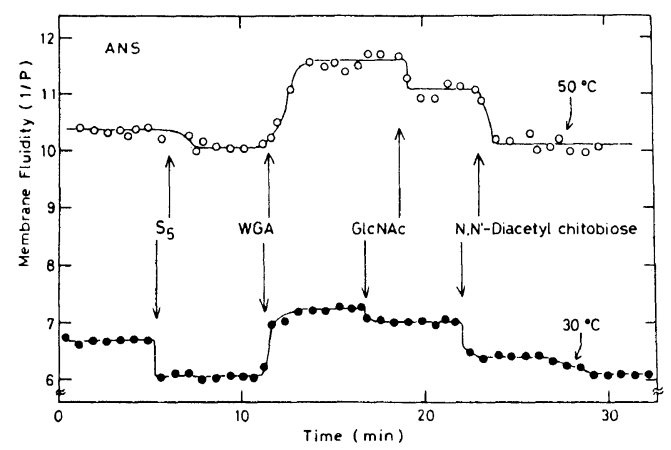

Figure 3. Membrane fluidity of DPPC vesicles monitored by ANS with various additives: $[\mathrm{DPPC}]=1.0 \mathrm{mM} ;\left[\mathrm{S}_{5}\right]=$ $1.89 \times 10^{-2} \mathrm{mM} ;[$ WGA $]=9.0 \times 10^{-3} \mathrm{mM}$; $[\mathrm{GlCNAC}]=5.9 \mathrm{mM} ;\left[\mathrm{N}, \mathrm{N}^{\prime}\right.$-diacetylchitobiose $=1.36 \mathrm{mM}$; [ANS $]=1.6 \times 10^{-1} \mathrm{mM}$.

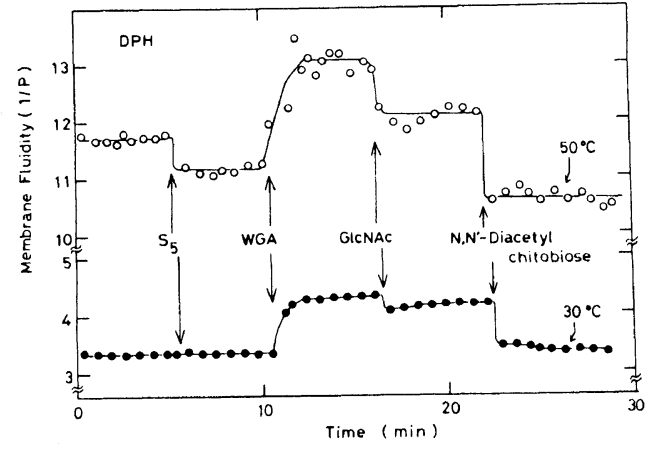

Figure 4. Membrane fluidity of DPPC vesicles monitored by DPH with various additives. The concentrations of the additives are the same as those in Figure 3 , and $[\mathrm{DPH}]=1.6 \times 10^{-2} \mathrm{mM}$.

core as monitored by $\mathrm{DPH}$ at $30^{\circ} \mathrm{C}$ did not decrease. The addition of WGA to $\mathrm{S}_{5}$-containing vesicles strongly increased the membrane fluidity. The action of WGA to the membrane was antagonized by GlcNAC only partly. A complete restoration of membrane fluidity was attained only by the addition of $\mathrm{N}, \mathrm{N}^{\prime}$-diacetylchitobiose after the dissociation of intervesicular aggregation. It was confirmed that the WGA effects on membrane fluidity were $S_{5}$-dependent.

Permeation of $\mathrm{CF}$ across the lipid membrane was examined with $\mathrm{CF}-$ trapped vesicles. CF leakage from vesicles was relatively intensive immediately after the addition of $S_{5}$ or $S_{10}$ to the suspension of vesicles and became moderate after a certain time lapse. This result shows that $\mathrm{S}_{5}$ or $\mathrm{S}_{10}$ perturbs the membrane structure to some extent at the time of insertion. The extent of membrane perturbation induced by the addition of glycopeptide increased in the order $\mathrm{S}_{5}<\mathrm{S}_{10}$. It was found in other experiments that the perturbation in membrane structure by the $\mathrm{S}_{5}$ or $\mathrm{S}_{10}$ distribution is enhanced by the complexation with WGA.

$\mathrm{S}_{5}$ - or $\mathrm{S}_{10} 0^{-c o n t a i n i n g ~} \mathrm{DPPC}$ vesicles did not show a voltage-dependent electric current, that is, the evidence of ion channel formation. It was considered that $\mathrm{S}_{5}$ and $\mathrm{S}_{10}$ do not meet the conditions for channel-forming species that the transmembrane region of intrinsic membrane proteins takes an $\alpha$-helical structure and the length of successive amino acid residues in the $\alpha$-helical conformation corresponds to the thickness of a lipid bilayer membrane. 6,7

Phospholipid vesicles containing Mal-15-Ala or Mal-25-Ala

$\alpha$-Helical oligoalanines having a maltose unit at one end of the chain and an 9-anthracenecarbonyl group at the other end were prepared. IR and $C D$ spectroscopy indicated that the peptide chain of the glycopeptides takes an $\alpha$-helical conformation both in a solid state and in solution.

The distribution of glycopeptides to the lipid bilayer membrane was investigated by fluorescence spectoscopy. In the presence of DMPC vesicles, Mal-15 and 25-Ala emitted stable fluorescence above the phase-transition temperature of the lipid membrane, indicating the distribution of the glycopeptides to the lipid bilayer membrane.

DPPC vesicles aggregated on adding Mal-15-Ala below the phase-transition temperature of the membrane, while they did not above the phase- 
(A)

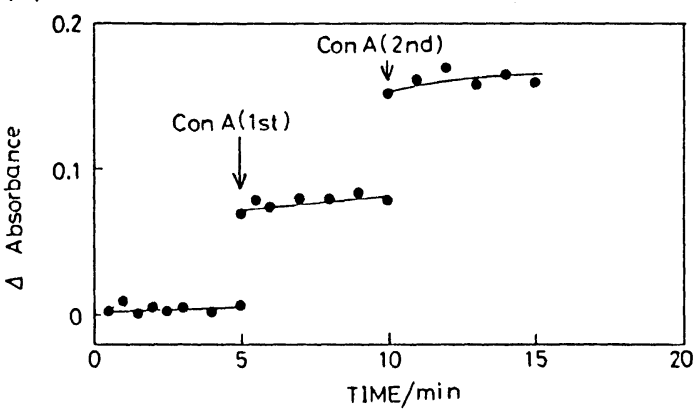

(B)

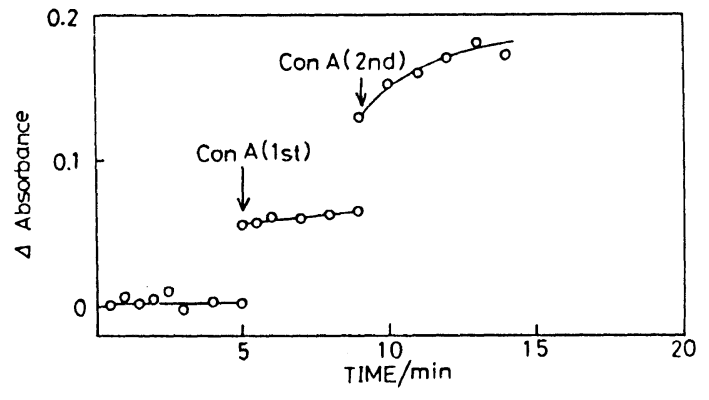

Figure 5 Con $A$-induced aggregation of DPPC vesicles containing Mal-15-Ala (A) or Mal-25-Ala (B) at $50^{\circ} \mathrm{C}$. [DPPC] $=1 \mathrm{mM}$; $[$ Mal-15 or $25-\mathrm{Ala}]=6.7 \times 10^{-4} \mathrm{mM}$; $[$ Con $\mathrm{A}]=1.7$ $\times 10^{-3} \mathrm{~mm}$ (at the $1 \mathrm{st}$ addition), $3.4 \times 10^{-3} \mathrm{~mm}$ (at the $2 \mathrm{nd}$ addition).

transition temperature. The addition of Ma1-25-Ala to DPPC vesicles below the phase-transition temperature did not induce the aggregation. However, the vesicles containing Mal-25-Ala aggregated, when the temperature was raised above the phase-transition temperature and then decreased below it. It was considered that Mal-25-Ala, when added below the phasetransition temperature, was loosely bound to the lipid membrane, but that upon raising temperature above the phase-transition temperature Mal-25Ala was incorporated into the lipid membrane. These experimental observations indicate that the glycopeptides are bound by the lipid bilayer membrane in a liquid-crystalline state so tightly that the membrane structure is destabilized to cause the aggregation of vesicles at lower temperatures than the phase-transition temperature.

The addition of Con A to DPPC vesicles containing Mal-15 or 25-Ala induced agglutination of vesicles as shown in Figure 5. In the absence of glycopeptides the turbidity change was not significant. Therefore, it was suggested that the glyco moiety of the glycopeptides is exposed to the aqueous phase, and the peptide chain is buried in the lipid membrane. However, the aggregated DPPC vesicles were not segregated by the addition of maltose. It was supposed that a tight aggregation occurred through
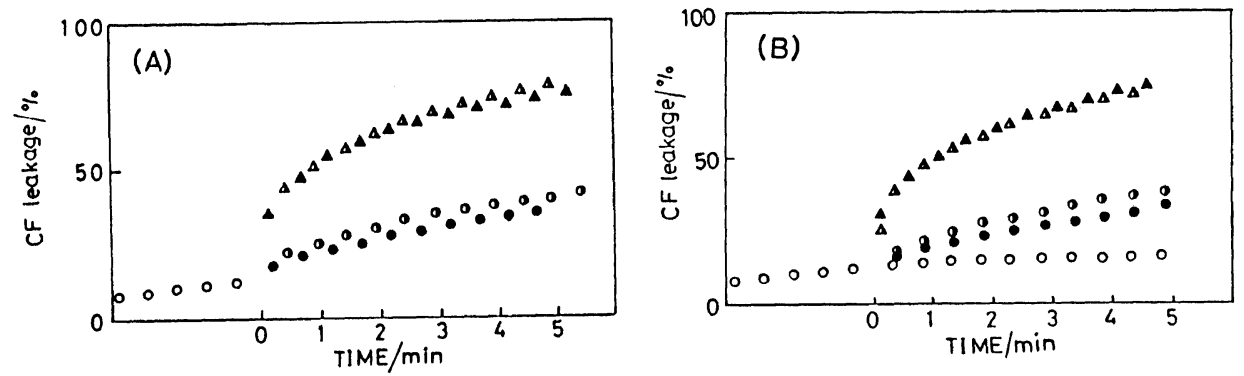

Figure 6. CF leakage from DPPC vesicles with the addition of Mal-15-Ala (A) and Mal-25-Ala (B), and the effect of Con A at $50^{\circ} \mathrm{C}$. [DPPC] $=3.1 \times 10^{-1} \mathrm{mM}$. 0 , without additives: $0,[\mathrm{Mal}-15$ or $25-\mathrm{Ala}]=1.6 \times 10^{-3} \mathrm{mM} ; 0,[\mathrm{Mal}-$ 15 or $25-\mathrm{Ala}]=1.6 \times 10^{-3} \mathrm{mM}$ and $[\mathrm{Con} \mathrm{A}]=4.0 \times 10^{-4} \mathrm{mM}$; $\Delta$, [Mal-15 or 25-Ala $]=8.0 \times 10^{-3} \mathrm{mM} ; \boldsymbol{A},[\mathrm{Mal}-15$ or $25-\mathrm{Ala}]=8.0 \times 10^{-3} \mathrm{mM}$ and $[\operatorname{Con} \mathrm{A}]=4.0 \times 10^{-4} \mathrm{mM}$. 
the interaction of dehydrated surface of the lipid membrane after Con A-induced preliminary association of vesicles.

Fluorescence quenching of Mal25-Ala by acrylamide in the presence of DMPC vesicles was less significant than in a buffer solution, indicating that the 9-anthracenecarbonyl group of the glycopeptide is buried in the hydrophobic core of the lipid bilayer membrane. The emission of Mal-15 and 25-Ala in the presence of DPPC vescles was quenched more efficiently by 16- [Ant-CO-Gly-OEt] $=2.4 \times 10^{-3} \mathrm{mM}$. doxylstearic acid than by 5-doxylstearic acid, implying that the $\mathrm{N}$-terminal region of the glycopeptides is located near the middle of the membrane.

The addition of glycopeptides to the suspension of CF-trapped vesicles enhanced the CF leakage from the vesicles as shown in Figure 6 . However, a simultaneous addition of Con $A$ and glycopeptide to CF-trapped vescles did not change the rate of $\mathrm{CF}$ leakage. It seems that these glycopeptides are not molecularly dispersed in the membrane, but are distributed by forming aggregates.

The excitation energy transfer from N-stearoyltryptophan to 9-anthracene carbonyl group of Mal-15 and 25-Ala in the presence of DMPC vesicles was measured and is summarized in Table I. Notably, the energytransfer efficiencies of Mal-15 and 25-Ala were lower than that of AntCO-Gly-OEt. Since the latter is supposed to be located near the membrane surface because of its low hydrophobicity, 5 the low energy-transfer efficiencies of glycopeptides indicate that the N-terminal of the glycopeptides is deeply incorporated into the hydrophobic core of the lipid membrane. The higher energy-transfer efficiency of Mal-25-Ala than that of Mal-15-Ala should indicate that the proportion of Mal-25-Ala residing near the membrane surface is higher than that of Mal-15-Ala.

Upon application of membrane potential by the addition of valinomy$\operatorname{cin}\left(1.6 \times 10^{-5} \mathrm{M}\right)$ to the suspension of egg yolk lecithin vesicles containing $0.1 \mathrm{M} \mathrm{KCl}$ in HEPES buffer containing $0.1 \mathrm{M} \mathrm{NaCl}$, the energy-transfer efficiency was slightly increased. If the macrodipole of glycopeptides interacts with the electric field generated across the membrane, the fraction of glycopeptides pointing perpendicularly to the membrane surface should increase, resulting in decreased energy-transfer efficiency. However, this was not the case. Taking these facts and literature reports together into account, the glycopeptides Mal-15-Ala and Mal-25-Ala should not necessarily take the transmembrane orientation spontaneously, though the peptide chain is long enough to span across the membrane.

Phospholipid vesicles containing alamethicin model peptides

As models for alamethicin that forms a voltage-dependent ion channel across the lipid bilayer membrane, sequential peptides containing $\alpha^{-}$ aminoisobutyric acid (Aib) residue, Boc-(Ala-Aib) ${ }_{n}-O M e(n=2,4,6,8)$, their derivatives carrying a fluorescent anthryl group at the $N$-terminal, Boc-Ser $\left(\mathrm{CH}_{2} \mathrm{Ant}\right)-(\mathrm{Ala}-\mathrm{Aib})_{n}-\mathrm{OMe}(\mathrm{n}=2, \mathrm{~A} 4 ; \mathrm{n}=4, \mathrm{~A} 8 ; \mathrm{n}=6, \mathrm{~A} 12 ; \mathrm{n}=8, \mathrm{~A} 16 ; \mathrm{n}=$ 10, A20), and their derivatives carrying a hydrophilic lactose unit at the C-terminal, Boc-(Ala-Aib) ${ }^{-L a c}(n=2, G 4 ; n=4, G 8)$, were prepared. It was expected that the incorporation of Aib units inhibits aggregation of 
peptides and stabilizes $\alpha$-helical structure of peptides in lipid bilayer membranes.

The conformation

and behaviors of the sequential peptides Boc$(\mathrm{Ala}-\mathrm{Aib})_{\mathrm{n}}$-OMe and $\mathrm{A} 2 \mathrm{n}$ in ethanol solution and in DMPC or DPPC vesicles were investigated by suitable methods; conformation in ethanol solution by CD spectroscopy, aggregation in HEPES buffer solution by intensities of monomer and excimer emission, disturbance of DPPC membrane structure upon incorporation by CF leakage, and change of DMPC membrane fluidity upon incorporation by fluorescence depolarization. These properties and behaviors of the sequential peptides are summarized in Table II in reference to the chain length of the peptides. The experimental results shown in Table II indicate that long-chain peptides (Ala-Aib) n are distributed to vesicles pointing perpendicularly to the lipid membrane with the C-terminal group on the surface and aggregate to form $\alpha$-helix bundle. This orientation of $(A l a-A i b)_{n}$ in lipid membrane would favor formation of ion channel upon application of electric potential across the membrane.

$$
\text { The apparatus }
$$

for the measurement of electric current across a black lipid membrane (BLM) is shown in Figure 7 . A teflon sheet of a thickness $0.25 \mu \mathrm{m}$ having a hole of a diameter $0.1-0.3 \mathrm{~mm}$ was coated with a $60 \%$ hexadecane solution in $\mathrm{n}$-hexane and fixed at the midale of an electric cell. Both chambers were filled with $\mathrm{KCl}$ solution ( 1

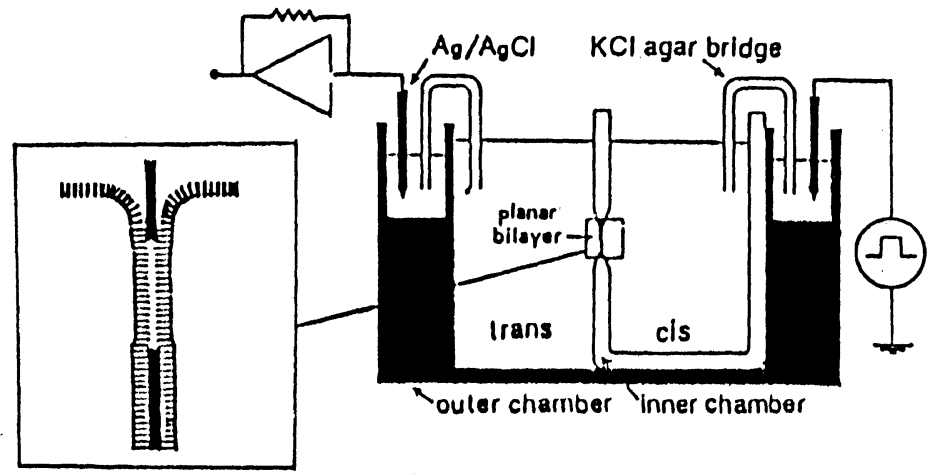

Figure 7. The apparatus for the measurement of electric current across lipid membrane.

M). A planar bilayer of azolectin was formed from a $n$-hexane solution $(0.1 \%)$ by the stick-on method. 8 Boc-(Ala-Aib) ${ }^{8}$-OMe $(n=4,8)$ was added to both sides of the azolectin bilayer membrane without breaking membrane and the current response to the electric potential applied was recorded. The current/ voltage relation of Boc-(Ala-Aib)n -OMe-containing azolectin BLM is shown in Figure 8. It was found that Boc-(Ala-Aib) n -OMe ( $n=4$ and 8 ) were able to form a voltage-dependent ion channel across the BLM, and that the channel-forming ability of the peptide having $n=8$ was larger than that of the peptide having $n=4$. The higher ability of $n=8$ peptide to form the voltage-dependent ion channel is explained in terms of the higher stability of $\alpha$-helix conformation and the higher tendency for aggregation.

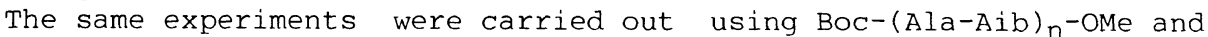
Boc-(Ala-Aib) $n-L a c$, and the results are shown in Figure 9. The glycopeptide showed a higher ability to form a voltage-dependent ion channel across the BLM than the peptide. The introduction of a hydrophilic sugar 


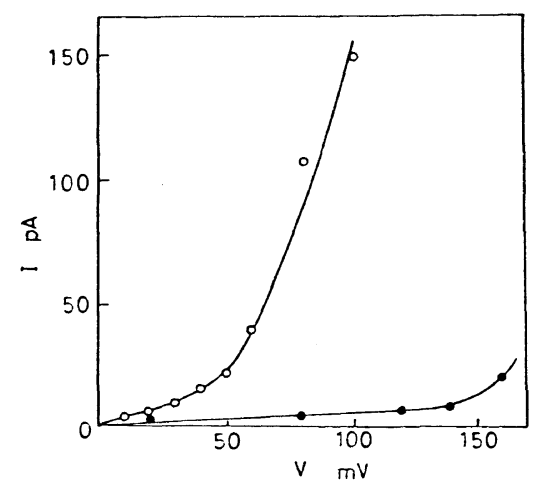

Figure 8. The current/voltage relationship of the azolectin membrane ( $0.3 \mathrm{~mm}$ diameter) con-

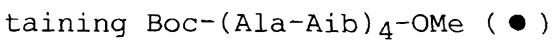
or Boc-(Ala-Aib) 8 -OMe (o) (3x $\left.10^{-3} \mathrm{mg} / \mathrm{ml}\right)$.

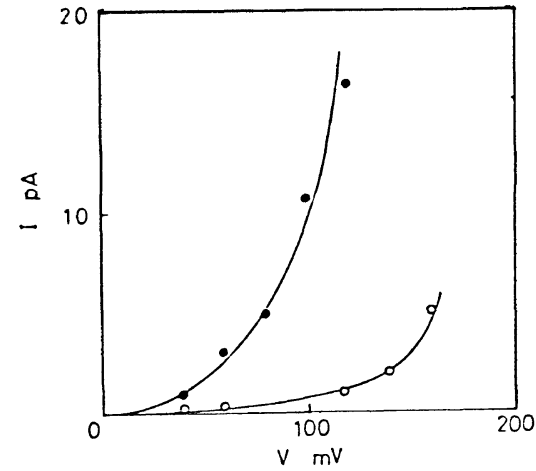

Figure 9. The current/voltage relationship of the azolectin membrane (0.1 mm diameter) containing $\mathrm{Boc}-(\mathrm{Ala}-\mathrm{Aib}) 4$-Lac or Boc-(Ala-Aib) $4^{-O M e ~(0) ~(3 x ~}$ $\left.10^{-3} \mathrm{mg} / \mathrm{ml}\right)$.

group at the $\mathrm{C}$ terminal yields amphiphilicity in the primary structure of the peptide and then leads to increased interactions with lipid membrane, resulting in the enhancement of channel-forming tendency.

A lectin RCA60, which is specific to lactose, was added to the Boc$(\mathrm{Ala}-\mathrm{Aib})_{\mathrm{n}}$-Lac-containing BLM, and voltage-dependent current was measured. The experimental results are shown in Figure 10. It is clearly shown in Figure 10 that the channel-forming ability of Boc-(Ala-Aib) $n$-Lac was increased in the presence of a specific lectin. The enhanced formation of ion channel could be ascribable to facililated formation and increased stability of the aggregate of $\alpha$-helical glycopeptides in the presence of lectin.
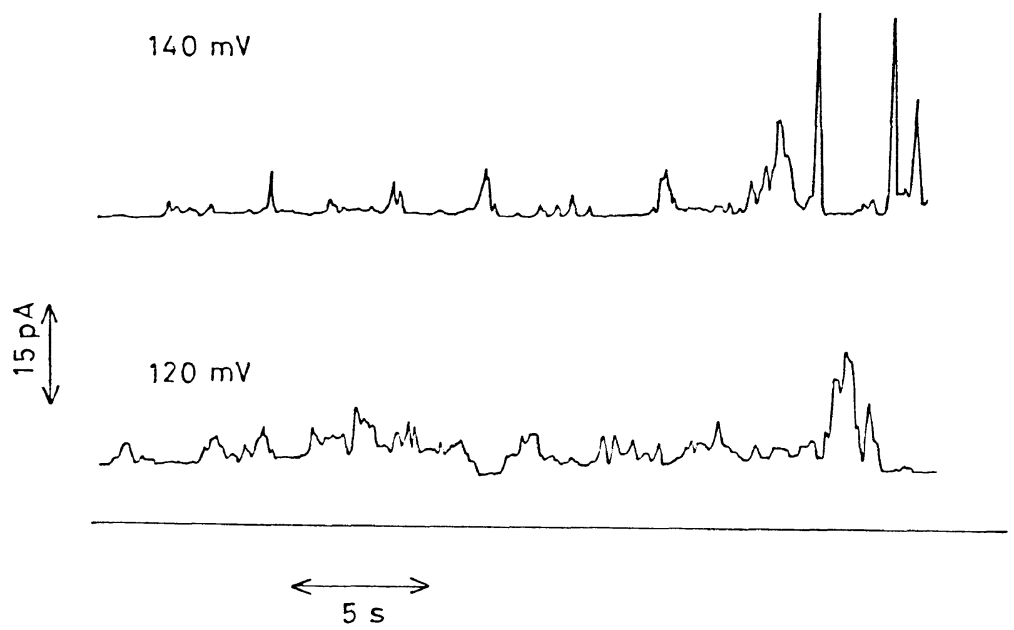

Figure 10. Formation of single ion-channel of Boc$(\mathrm{Ala}-\mathrm{Aib}) 4^{-\operatorname{Lac}}\left(3 \times 10^{-3} \mathrm{mg} / \mathrm{ml}\right)$ across the azolectin membrane ( $0.1 \mathrm{~mm}$ diameter) with the application of $140 \mathrm{mV}$ potential and the effect of RCA 60 addition ( $1.25 \times 10^{-2} \mathrm{mg} / \mathrm{ml}$ ) under the electric potential (120 $\mathrm{mV})$. 


\section{Glycopeptides in Lipid Vesicles as Receptor Model}

\section{REFEREIJCES}

1. T. Ohtoyo, M. Shimagaki, K. Otoda, S. Kimura, and Y. Imanishi, Biochemistry, 27, 6458 (1988)

2. K. Otoda, S. Kimura, and Y. Imanishi, Bull. Chem. Soc. Jpn., 63, 489 (1990)

3. K. Otoda, S. Kimura, and Y. Imanishi, Polym. Prepr., Jpn., 39, 849 (1990)

4. J. Barbet, P. Machy, A. Truneh, and L. D. Leserman, Biochem. Biophys. Acta, 772, 347 (1984)

5. A. Uemura, S. Kimura, and Y. Imanishi, Biochem. Biophys. Acta, 729, 28 (1983)

6. P. Argos, J. K. M. Rao, and P. A. Hargrave, Eur. J. Biochem., 128, 565 (1982)

7. D. Eisenberg, Ann. Rev. Biochem., 53, 595 (1984)

8. M. Montal and P. Müller, Proc. Natl. Acad. Sci. USA, 69, 3561 (1971) 